\title{
Compressive sensing in distributed radar sensor networks using pulse compression waveforms
}

\author{
Lei $\mathrm{Xu}^{{ }^{*}}$, Qilian Liang ${ }^{1}$, Xiuzhen Cheng ${ }^{2}$ and Dechang $C h e n^{3}$
}

\begin{abstract}
Inspired by recent advances in compressive sensing (CS), we introduce CS to the radar sensor network (RSN) using pulse compression technique. Our idea is to employ a set of stepped-frequency (SF) waveforms as pulse compression codes for transmit sensors, and to use the same SF waveforms as the sparse matrix to compress the signal in the receiving sensor. We obtain that the signal samples along the time domain could be largely compressed so that they could be recovered by a small number of measurements. A diversity gain could also be obtained at the output of the matched filters. In addition, we also develop a maximum likelihood (ML) algorithm for radar cross section (RCS) parameter estimation and provide the Cramer-Rao lower bound (CRLB) to validate the theoretical result. Simulation results show that the signal could be perfectly reconstructed if the number of measurements is equal to or larger than the number of transmit sensors. Even if the signal could not be completely recovered, the probability of miss detection of target could be kept zero. It is also illustrated that the actual variance of the RCS parameter estimation $\widehat{\theta}$ satisfies the CRLB and our ML estimator is an accurate estimator on the target RCS parameter.
\end{abstract}

Keywords: Compressive sensing, Radar sensor networks, Pulse compression, Stepped-frequency waveform, Target RCS

\section{Introduction}

Current requirements in warfighting functionality result in obtaining accurate and timely information about battlespace objects and events so that the warfighters can make decision about reliable location, tracking, combat identification and targeting information. While massive amounts of data will be generated by a penetrating sensor, it is important for the warfighters to find technologies that not only integrate information from diverse sources but also provide indications of information significance in ways that help them to make tactical decision. The RCS is the property of a scattering object, or target, which represents the magnitude of the echo signal returned to the radar by the target. Hence, we could have different classes with different RCS values representing corresponding targets, such as bird, conventional unmanned winged missile, small single-engine aircraft and large flight

\footnotetext{
*Correspondence: xu@wcn.uta.edu

1 Department of Electrical Engineering, University of Texas at Arlington, Arlington, TX 76010, USA

Full list of author information is available at the end of the article
}

aircraft. In this article, we will study the target RCS in a radar sensor network (RSN) by using compressive sensing techniques.

It is well known that wireless sensor networks (WSN) are a fast growing class of systems. In [1], the authors presented a new method that makes use of the properties of data of multiple sensors to enable reliable data collection. In [2], the authors adopted a mutual-informationbased sensor selection (MISS) algorithm to help sensing devices collaborate among themselves to improve the target localization and tracking accuracies. Alike WSN, RSN has been recently considered to overcome the performance degradation of a single radar. In [3], the authors design a network of distributed radar sensors that work in an ad hoc fashion and the simulation results showed that proposed RSN can provide much better detection performance than that of single radar sensor. However, RSN is quite different from WSN. The waveform of each radar sensor has to be properly designed, otherwise, these radar sensors are likely to badly interfere with each other in the RSN. As a result, the design of radar

\section{它 Springer}

(c) 2013 Xu et al: licensee Springer. This is an Open Access article distributed under the terms of the Creative Commons

Attribution License (http://creativecommons.org/licenses/by/2.0), which permits unrestricted use, distribution, and reproduction in any medium, provided the original work is properly cited. 
waveforms has been under the study with the goal of optimizing the performance of the RSN. In [4], binary coded pulses using simulated annealing in RSN are proposed. Liang [5] studied constant frequency (CF) pulse waveform design and proposed Maximum-Likelihood (ML) automatic target recognition (ATR) approach for both nonfluctuating and fluctuating targets in a network of multiple radar sensors. Furthermore, Liang [6] studied the RSN design based on linear frequency modulation (LFM) waveforms and also applied the LFM waveforms to RSN in the context of ATR with delay-Doppler uncertainty. In addition, it is known that the pulse compression technique allows a radar to achieve both the energy of a long pulse and the resolution of a short pulse, without the high peak power which is required by a high energy short duration pulse [7]. Pulse compression waveforms are obtained by adding frequency or phase modulation to a simple pulse. A stepped-frequency (SF) waveform is a frequency modulation waveform for obtaining a large bandwidth, and thus a fine range resolution without requiring intrapulse frequency modulation. The most common SF waveform employs a linear frequency stepping pattern, where the RF frequency of each pulse is increased by $\Delta F$. This representation motivates the applicability of the recently proposed compressive sensing (CS) theory $[8,9]$ that refers to such signals as 'sparse' or 'compressible.'

Recent results in compressive sensing have shown that the information from a signal may be captured with a small set of nonadaptive, linear measurements as long as the signal is sparse in some basis or frame [10-12]. If the signal is properly chosen, the number of measurements to recover the signal can be much smaller than the number of Nyquist-rate samples. The application of compressive sensing to radar imaging has been investigated in $[13,14]$. A CS-based data acquisition and imaging method was proposed to study a number of point-like targets for SF continuous wave ground penetrating radars (SFCW-GPRs) in [15]. In [16], the authors proposed the step-frequency with compressive sampling (SFR-CS), that achieves high target range and speed resolution using significantly smaller bandwidth than transitional step-frequency radar. The application of CS to multiple input and multiple output (MIMO) radar has also attracted a lot of attention in recent research. The study of [17] studied angle-Doppler estimation of multiple targets for MIMO radar system. In addition, the authors in [18] also considered the range estimation performance of their proposed method for MIMO radar systems that employ CS. Based on adaptive radar design, the authors in [19] studied MIMO radar with widely separated antennas in the context of sparse modeling for estimating the positions and velocities of multiple targets.
Due to the expansion of data introduced to RSN, the compression and reconstruction of the received data is a design challenge of future RSN. Unlike the above research, the study in this article explores how to exploit compressive sensing in RSN composed of a number of transmit sensors but only one receiving sensor. It is known that the signal must be 'compressible' for compressive sensing to have benefit. Recognizing that the SF train could act as the sparsity basis for the signal, we apply it as a pulse compression code to construct the 'compressible signal' for a transmit sensor. We choose the Gaussian matrix as the measurement matrix that satisfies the restricted isometry property (RIP) with this basis. However, there are still a number of challenges in most CS theory applied to radar which specifically mentions that it eliminates the need for matched filter in the radar receiving sensor. In [13], only the range parameter of the target was studied and the target reflectivity being probed must be compressible in some basis before their CS-based radar system could work. In [20], the transmitted signal must be sufficiently 'incoherent' and the targets have to be radially aligned with the transmitter and receiver. In this article, we propose and investigate a totally different model of CS-based RSN system. Hence, the matched filters are still used in the receiving sensor.

After applying compressive sensing to RSN, we also perform target RCS value estimation. We propose an ML algorithm to estimate the target RCS parameter and use the Cramer-Rao lower bound (CRLB) to validate our theoretical result. In the simulation parts, the performance of signal recovery and the performance of target detection are studied as well as the performance of target RCS value estimation. The simulation results show that the signal could be precisely recovered if the number of measurements is no less than the number of sensors in RSN. The target could be perfectly detected even if the signal could not be precisely recovered. As a result, much smaller measurement matrix could be used on the receive part for the purpose of target detection. Finally, the actual variance of the RCS parameter estimation $\widehat{\theta}$ satisfies the CRLB.

The article is organized as follows. In Section 2, we present our basic model by applying CS to RSN exploiting the pulse compression technique. In Section 3, we show that the range resolution is increased for RSN using Step-Frequency waveforms as the pulse compression codes. In Section 4, we propose a Maximum Likelihood (ML) algorithm for target RCS value estimation and derive the CRLB accordingly. In Section 5, the simulation results illustrate the performance of signal recovery after decompression, target detection and target RCS value estimation. Finally, conclusions are drawn in Section 6. 


\section{The basic model}

\subsection{The produced signal for compressed sensing}

The SF pulse train is a pulse burst waveform which obtains large overall bandwidth while maintaining narrow instantaneous bandwidth. Each pulse in the burst is a simple, constant-frequency pulse; however, the RF frequency is added by a frequency step $\Delta F$ between consecutive pulses. The most common SF waveform employs a linear frequency stepping pattern, where the RF frequency of each pulse is increased by $\Delta F$ Herts from the preceding pulse. In addition, the frequency steps can be added to a train of unmodulated pulses, as well as to a train of modulated pulses. The SF waveform we study in this article is expressed as follows:

$$
s(t)=\sum_{i=0}^{I-1} s_{i}\left(t-i T_{p}\right) e^{j 2 \pi i \Delta F\left(t-i T_{p}\right)},
$$

where $T_{p}$ is the pulse repetition interval and $I$ is the number of pulses in a burst (train) of pulses. Observing the right side of Equation (1), we see that $e^{j 2 \pi i \Delta F\left(t-i T_{p}\right)}$ can be expressed into the orthogonal Fourier basis $e^{j 2 \pi \frac{n i}{I}\left(t-i T_{p}\right)}$, where $\Delta F=\frac{n}{I}$ and $n$ is the index of a transmit sensor in a RSN. Here, we let the frequency step $\Delta F$ among each pulse burst waveform corresponding to each specific transmit sensor be different and related to the index $n$. The goal of CS is to perform good reconstruction of the signal by using a few sparse samples. Here, we construct the data in such a way that is compressed already. We establish a RSN which uses the SF waveforms as pulse compression codes. The RSN consists of $N$ transmit sensors indexed by $n$ and only one receiving sensor. Then $s_{n}(t)=\sum_{i=0}^{I-1} x_{n}\left(t-i T_{p}\right) e^{j 2 \pi \frac{n i}{I}\left(t-i T_{p}\right)}$ denotes the transmit signal for the transmit sensor $n$. Let $x_{n}(t)=\sum_{i=0}^{I-1} x_{n}\left(t-i T_{p}\right)$ and $\psi_{n}(t)=\sum_{i=0}^{I-1} e^{j 2 \pi \frac{n i}{I}\left(t-i T_{p}\right)}$ denote the information signal and the pulse compression waveforms, where $N$ is the number of transmit sensors and $I$ is still the number of pulses in a burst. In order for the later use, we express the preceding equations in vector format that $\bar{x}_{n}=\left[x_{n}\left(t_{1}\right), x_{n}\left(t_{2}\right), \ldots, x_{n}\left(t_{I}\right)\right]^{T}$ and $\bar{\psi}_{n}=$ $\left[e^{j 2 \pi \frac{n}{I} t_{1}}, e^{j 2 \pi \frac{2 n}{I} t_{2}}, \ldots, e^{j 2 \pi \frac{n I}{I} t_{I}}\right]^{T}=\left[\psi_{1 n}, \psi_{2 n}, \ldots, \psi_{I n}\right]^{T}$.

Then, $S=\Psi X^{T}$ is the transmit matrix, where $X=\left[\bar{x}_{1}, \bar{x}_{2}, \ldots, \bar{x}_{N}\right]$ and $\Psi=\left[\bar{\psi}_{1}, \bar{\psi}_{2}, \ldots, \bar{\psi}_{N}\right]$.

The RCS is the property of a scattering target that is included in the radar equation to represent the magnitude of the echo signal returned to the radar by the target. As a result, the RCS returned to the receiving sensor by the target is assumed to have isotropic reflectivity modeled by zero-mean, unit-variance, independent and identically distributed (i.i.d.) Gaussian complex random variables $\lambda_{n}$. Assuming that it is the slow fading system, where the amplitude and phase change imposed by the channel can be considered roughly constant over the period of use, we suppose that the Rayleigh distortion does not fluctuate during the whole pulse burst. The RCS can be modeled by the diagonal matrix

$$
\Sigma=\frac{1}{\sqrt{2 N}}\left[\begin{array}{cccc}
\lambda_{1} & 0 & \cdots & 0 \\
0 & \lambda_{3} & \ddots & \vdots \\
\vdots & \ddots & \ddots & 0 \\
0 & \ldots & 0 & \lambda_{N}
\end{array}\right]
$$

where the normalization factor makes the target average RCS $=\frac{\sum_{n=1}^{N}\left|\lambda_{n}\right|^{2}}{2 N}=1$ independent of the number of transmit sensors in the model. A popular method for representing the fluctuations of targets are the four statistical models described by Swerling in [21]. The nonfluctuating target modeled using non-zero constants for $\lambda_{n}=\lambda$ is identified as 'Swerling 0' or 'Swerling 5' model. For the fluctuating target, if $\left|\lambda_{n}\right|$ is drawn from the Rayleigh P.D.F. and varies independently from path to path, the target model represents a classical 'Swerling 2' model.

Taken all the above parameters into account, we use the following block diagram illustrating as Figure 1 to show our basic model.

A vector form of the received signal including the useful signal and the system noise is $\bar{r}$ and we will process with this $\bar{r}$ in the following section

$$
\begin{aligned}
\bar{r}=\operatorname{diag}\left(\Psi \Sigma X^{T}\right) & \left(\left[\begin{array}{llll}
\psi_{1,1} & \psi_{1,2} & \ldots & \psi_{1, N} \\
\psi_{2,1} & \psi_{2,2} & \ldots & \psi_{2, N} \\
\vdots & \vdots & \ddots & \vdots \\
\psi_{I, 1} & \psi_{I, 2} & \ldots & \psi_{I, N}
\end{array}\right] \cdot\left[\begin{array}{llll}
\lambda_{1} & 0 & \cdots & 0 \\
0 & \lambda_{2} & \ddots & \vdots \\
\vdots & \ddots & \ddots & 0 \\
0 & \ldots & 0 & \lambda_{N}
\end{array}\right]\right. \\
& \left.\cdot\left[\begin{array}{llll}
x_{1}\left(t_{1}\right) & x_{1}\left(t_{2}\right) & \ldots & x_{1}\left(t_{I}\right) \\
x_{2}\left(t_{1}\right) & x_{2}\left(y_{2}\right) & \cdots & x_{2}\left(t_{I}\right) \\
\vdots & \vdots & \ddots & \vdots \\
x_{N}\left(t_{1}\right) & x_{N}\left(t_{2}\right) & \ldots & x_{N}\left(t_{I}\right)
\end{array}\right]\right)+\bar{n}
\end{aligned}
$$

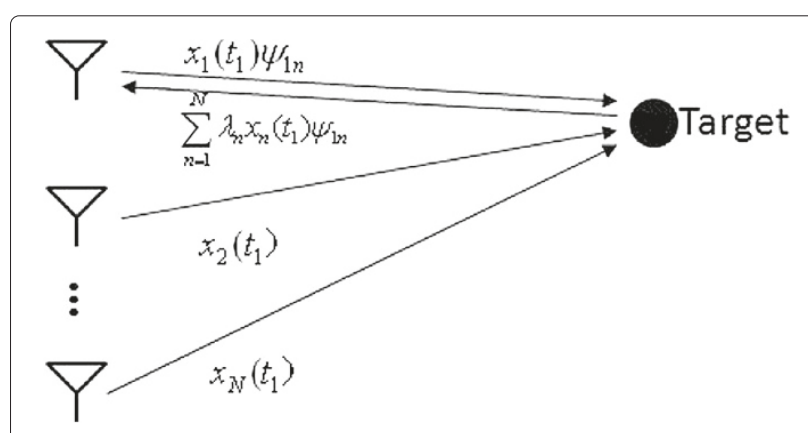

Figure 1 The block diagram of the model. 


$$
\begin{gathered}
=\frac{1}{\sqrt{2 N}}\left[\sum_{n=1}^{N} \lambda_{n} x_{n}\left(t_{1}\right) \psi_{1, n}, \sum_{n=1}^{N} \lambda_{n} x_{n}\left(t_{2}\right) \psi_{2, n}, \ldots,\right. \\
\left.\sum_{n=1}^{N} \lambda_{n} x_{n}\left(t_{I}\right) \psi_{I, n}\right]^{T}+\bar{n}
\end{gathered}
$$

where $\bar{n}$ is the noise vector.

\subsection{Decomposition and recovery of the signal}

In order to obtain some benefit form CS, we choose the same orthogonal basis $\bar{\psi}_{n}$ as the sparse matrix $\Psi$ to study the received signal $\bar{r}$ including the useful signal and the noise, where

$$
\bar{r}=\sum_{n=1}^{N} \theta_{n} \bar{\psi}_{n}=\Psi \bar{\theta}
$$

with $\bar{\theta}=\left[\theta_{1}, \ldots, \theta_{N}\right]^{T}=\left[<\bar{r}, \bar{\psi}_{1}>, \ldots,<\bar{r}, \bar{\psi}_{N}>\right]^{T}$. The CS can only work when there are $K$ non-zero coefficients in the vector $\bar{\theta}$, where $K<I$. According to the concepts of CS, there should be only a few large coefficients and many small ones in $\bar{\theta}$.

The CS approach uses a set of measurement probes $\left\{\bar{\varphi}_{m}=\left[\varphi_{1, m}, \varphi_{2, m}, \ldots, \varphi_{I, m}\right]^{T}\right\}_{m=1}^{M}$, where $M$ is significantly less than the dimensionality $I$ of each individual probe ( $I$ is just the size of receive signal $\bar{r}$ in our case). In a different way, we would like to recover all the $I$ coefficients of $\bar{r}$ by observing or measuring a subset $M$ of these

$$
y_{m}=<\bar{r}, \bar{\varphi}_{m}>, \forall m \in M,
$$

where $\mathbf{Y}=\left[y_{1}, y_{2}, \ldots, y_{M}\right]^{T}, M \subset 1, \ldots, I$. Since random matrix $\Phi$ with independent identically distributed (i.i.d.) entries, e.g., Gaussian or binary entries, exhibit a very low coherence with any fixed sparse matrix $\Psi$ [13], we take the Gaussian matrix as the measurement matrix.

The recovery of $\bar{r}$ is done by solving a constrained $l_{1}$ norm minimization problem and the reconstructed $\bar{r}^{*}$ is given by $\bar{r}^{*}=\Psi \bar{\theta}^{*}$, where $\bar{\theta}^{*}$ is the solution to the convex optimization program

$$
\min _{\bar{\theta}^{*} \in R^{I}}\left\|\bar{\theta}^{*}\right\|_{l_{1}} \text { subject to } y_{m}=<\bar{\varphi}_{m}, \Psi \bar{\theta}^{*}>, \forall m \in M
$$

We choose the solution whose coefficient sequence has the minimal $l_{1}$ norm.

We describe how our model recovers a particular $\bar{r}^{*}$ in section 2.3. We first produce the transmit signal and receive it as $\bar{r}$. Then, we compute $\mathbf{Y}$ by compressing the received signal $\bar{r}$. Finally, we use the $l_{1}$ minimization with relaxed constraints to reconstruct the original signal $\bar{r}^{*}$.

\subsection{The basic model}

Input: $\Phi, \Psi, \Sigma, X$

$\bar{r} \leftarrow \bar{r}=\operatorname{diag}\left(\Psi \Sigma X^{T}\right)$

$\mathbf{Y} \leftarrow y_{m}=<\bar{\varphi}_{m}, \Psi \bar{\theta}>$, for all $m \in M$

$$
\begin{aligned}
& \bar{\theta}^{*} \leftarrow \min _{\bar{\theta}^{*} \in R^{I}}\left\|\bar{\theta}^{*}\right\|_{l_{1}} \text {, subject to } \\
& \left\|\Phi^{T} \Psi \bar{\theta}^{*}-y_{m}\right\|_{l_{2}} \leq \epsilon \\
& \text { Output: } \bar{r}^{*}=\Psi \bar{\theta}^{*}
\end{aligned}
$$

We explicitly tie together the parameters for the decompressive process, i.e.,

$$
\mathbf{Y}=\Phi^{T} \bar{r}^{*}=\Phi^{T} \Psi \bar{\theta}^{*}=A \bar{\theta}^{*}
$$

One of the important properties that $A=\Phi^{T} \Psi$ should satisfy is the restricted isometry property (RIP) [11]. A sufficient condition for the RIP is that the measurement vectors and the sparsity basis must be incoherent with each other. The coherence between the measurement matrix $\Phi$ and the sparsity basis $\Psi$ here is defined as

$$
\mu(\Phi, \Psi)=\max _{1 \leq m \leq M, 1 \leq n \leq N}\left|<\varphi_{m}, \psi_{n}>\right|
$$

If $\Phi$ and $\Psi$ contain uncorrelated elements, the coherence is large. So the 'incoherency' here means that the inner products between the probes and the sparsity basis vectors are small, or, in other words, $\mu$ is small.

In this article, we express the SF coefficients in the method of Fourier basis as the sparse matrix

$\bar{\psi}_{n}=\left[e^{j 2 \pi \frac{n}{I} t_{1}}, e^{j 2 \pi \frac{2 n}{I} t_{2}}, \ldots, e^{j 2 \pi \frac{n I}{I} t_{I}}\right]^{T}=\left[\psi_{1 n}, \psi_{2 n}, \ldots, \psi_{I n}\right]^{T}$

Here, the sparse matrix can be non-squared, i.e., the number of rows can be different from the number of columns in the sparse matrix. The number of rows is based on the number of transmit sensors $N$ and the number of columns depends on the number of time samples $I$. The reason why we can use such a non-squared matrix is that we use the matrix same as the sparse matrix to produce the 'compressible' transmit signal. In addition, the random waveforms $\varphi_{m}(t)$ with independent identically distributed (i.i.d) vectors are largely incoherent with the fixed basis $\Psi$. Hence, we choose the complex Gaussian random vectors as the measurement matrix in our study.

\subsection{The output of the matched filter}

Different from previous CS-based radar system, we proposed a totally new model for CS-based RSN. Since we use the matched filters in the receiving sensor, let us assume that the received signal could be well recovered as $\bar{r}^{*}$

$$
\begin{gathered}
\bar{r}^{*}=\bar{r}=\frac{1}{\sqrt{2 N}}\left[\sum_{n=1}^{N} \lambda_{n} x_{n}\left(t_{1}\right) \psi_{1, n}, \sum_{n=1}^{N} \lambda_{n} x_{n}\left(t_{2}\right) \psi_{2, n}, \ldots,\right. \\
\left.\sum_{n=1}^{N} \lambda_{n} x_{n}\left(t_{I}\right) \psi_{I, n}\right]^{T}+\bar{n}^{\prime},
\end{gathered}
$$

where $\bar{n}^{\prime}=\left[n_{1}^{\prime}, n_{2}^{\prime}, \ldots, n_{I}^{\prime}\right]^{T}$ is the noise vector. Actually, the power of noise $\bar{n}^{\prime}$ in the recovered signal is less than the system noise in the received signal in Equation (3). Since the $\bar{n}$ may not have good sparsity property in 
the basis $\Psi$, some information of $\bar{n}$ could not be exactly recovered by $\bar{n}^{\prime}$ Here, we choose the matched filters corresponding to the transmit signal $S=\Psi X^{T}$. Processing by a bank of matched filters, we express the output of the matched filter as the following matrix operations

$$
\begin{aligned}
& \bar{Z}=\left[\begin{array}{llll}
x_{1}^{*}\left(t_{1}\right) \psi_{1,1}^{*} & x_{1}^{*}\left(t_{2}\right) \psi_{2,1}^{*} & \cdots & x_{1}^{*}\left(t_{I}\right) \psi_{I, 1}^{*} \\
x_{2}^{*}\left(t_{1}\right) \psi_{1,2}^{*} & x_{2}^{*}\left(t_{2}\right) \psi_{2,2}^{*} & \cdots & x_{2}^{*}\left(t_{I}\right) \psi_{I, 2}^{*} \\
\vdots & \vdots & \ddots & \vdots \\
x_{N}^{*}\left(t_{1}\right) \psi_{1, N}^{*} & x_{N}^{*}\left(t_{2}\right) \psi_{2, N}^{*} & \cdots & x_{2}^{*}\left(t_{I}\right) \psi_{I, N}^{*}
\end{array}\right] \\
& \cdot \frac{1}{\sqrt{2 N}}\left[\begin{array}{l}
\sum_{n=1}^{N} \lambda_{n} x_{n}\left(t_{1}\right) \psi_{1, n}+\sqrt{2 N} n_{1} \\
\sum_{n=1}^{N} \lambda_{n} x_{n}\left(t_{2}\right) \psi_{2, n}+\sqrt{2 N} n_{2} \\
\vdots \\
\sum_{n=1}^{N} \lambda_{n} x_{n}\left(t_{I}\right) \psi_{I, n}+\sqrt{2 N} n_{I}
\end{array}\right] \\
& =\frac{1}{\sqrt{2 N}}\left[\sum_{n=1}^{N} \lambda_{n}\left[\sum_{i}^{I} x_{n}\left(t_{i}\right) \psi_{i, n} x_{1}^{*}\left(t_{i}\right) \psi_{i, 1}^{*}\right]+n_{1}^{\prime}\right. \text {, } \\
& \sum_{n=1}^{N} \lambda_{n}\left[\sum_{i}^{I} x_{n}\left(t_{i}\right) \psi_{i, n} x_{2}^{*}\left(t_{i}\right) \psi_{i, 2}^{*}\right]+n_{2}^{\prime}, \ldots \text {, } \\
& \left.\sum_{n=1}^{N} \lambda_{n}\left[\sum_{i}^{I} x_{n}\left(t_{i}\right) \psi_{i, n} x_{N}^{*}\left(t_{i}\right) \psi_{i, N}^{*}\right]+n_{N}^{\prime}\right]^{T}
\end{aligned}
$$

Since $\Psi$ is the Fourier basis in this article, and $X$ is information bits expressed as pulsed signals, it is easy to obtain that

$$
\sum_{i}^{I} x_{n}\left(t_{i}\right) \psi_{i, n} x_{k}^{*}\left(t_{i}\right) \psi_{i, k}^{*}= \begin{cases}E, & n=k \\ 0, & \text { Otherwise }\end{cases}
$$

where $E$ is the energy of the burst of pulses. Therefore,

$$
\bar{Z}=\frac{1}{\sqrt{2 N}}\left[\lambda_{1} E+n_{1}^{\prime}, \lambda_{2} E+n_{2}^{\prime}, \ldots, \lambda_{N} E+n_{N}^{\prime}\right]^{T}
$$

where $\lambda_{n}$ is the radar cross section for $n$th transmit sensor. According to the Equation (12), all the $N$ radar cross section parameters could be taken into account to detect or recognize the target, so that the diversity gain of transmit side can be obtained as well.

\section{Increased range resolution}

The range resolution improvement is one of the important properties of the linear SF waveforms (LSFWs). The details of the Doppler response and range resolution can be expressed by the ambiguity function (AF) of the LSFW [22]. The AF is defined as

$$
\widehat{A}(\tau, v) \equiv|\chi(\tau, v)|=\left|\int_{-\infty}^{\infty} u(t) u^{*}(t+\tau) \exp (j 2 \pi v t) d t\right|
$$

We designate the complex envelope of the SF pulse train by $u(t)$ given by

$$
u(t)=\sum_{m=0}^{M-1} u_{1}\left(t-m T_{p}\right) e^{j 2 \pi m \Delta F\left(t-m T_{p}\right)},
$$

where $u_{1}(t)$ is a radar pulse waveform. Figure 2 presents a sample AF of a SF train of unmodulated pulses calculated by using the MATLAB tool.

It is obvious to see that the range resolution of the signal is improved, but there are still prominent sidelobes in delay and ambiguity in Doppler. As a result, LFMs and SF can be combined to mitigate the raging lobes, i.e., SF train of LFM pulses could be used. An example of SF train of LFM is shown in Figure 3.

We compare the AF of a SF train of LFM pulses, as shown in Figure 3, with the AF of a SF train of unmodulated pulses as seen in Figure 2. Clearly, by adding the LFM, the range and the Doppler resolutions are improved by canceling the sidelobes along the delay and Doppler axes. As a result, we choose to use the SF train of LFM pulses as the transmit waveforms in our model to obtain both the range and Doppler resolution gain.

\section{Target RCS value estimation}

In this section, we use the maximum-likelihood (ML) estimation algorithm to perform target radar cross section (RCS) parameter estimation [23] in the proposed RSN model. For the 'Swerling 2' model, the RCS voltage $|\lambda(u)|$ follows a Rayleigh distribution and the I and Q subchannels of $\lambda(u)$ follow zero-mean complex Gaussian distribution with a variance $\gamma^{2}$ (the RCS average power value)

$$
\lambda(u)=\lambda_{I}(u)+j \lambda_{Q}(u)
$$

In addition, $n(u)=n_{I}(u)+j n_{Q}(u)$ follows a zero-mean complex Gaussian distribution with a variance $\sigma^{2}$ for each I and Q subchannel. We express Equation (12) as following

$\bar{Z}(u)=\left[\lambda_{1}(u) E+n_{1}^{\prime}(u), \lambda_{2}(u) E+n_{2}^{\prime}(u), \ldots, \lambda_{N}(u) E+n_{N}^{\prime}(u)\right]^{T}$

Here, we let

$$
\left|Z_{n}(u)\right|=\left|\lambda_{n}(u) E+n_{n}^{\prime}(u)\right|
$$

Since $\lambda_{n}(u)$ and $n_{n}^{\prime}(u)$ are zero-mean complex Gaussian random variables, $\lambda_{n}(u)+n_{n}^{\prime}(u)$ is zero-mean complex Gaussian random variable with a variance $E^{2} \gamma^{2}+\sigma^{2}$.

Assuming that $y_{n} \cong\left|Z_{n}(u)\right|$ follows a Rayleigh distribution:

$$
f\left(y_{n}\right)=\frac{y_{n}}{E^{2} \gamma^{2}+\sigma^{2}} \exp \left[-\frac{y_{n}^{2}}{2\left(E^{2} \gamma^{2}+\sigma^{2}\right)}\right]
$$

Let $\mathbf{y}=|\bar{Z}(u)|,|\bar{Z}(u)|=\left[\left|Z_{1}(u)\right|,\left|Z_{2}(u)\right|, \ldots,\left|Z_{N}(u)\right|\right]$ and $y_{n}=\left|Z_{n}(u)\right|$, we can obtain that $\mathbf{y} \cong\left[y_{1}, y_{2}, \ldots, y_{N}\right]$. 


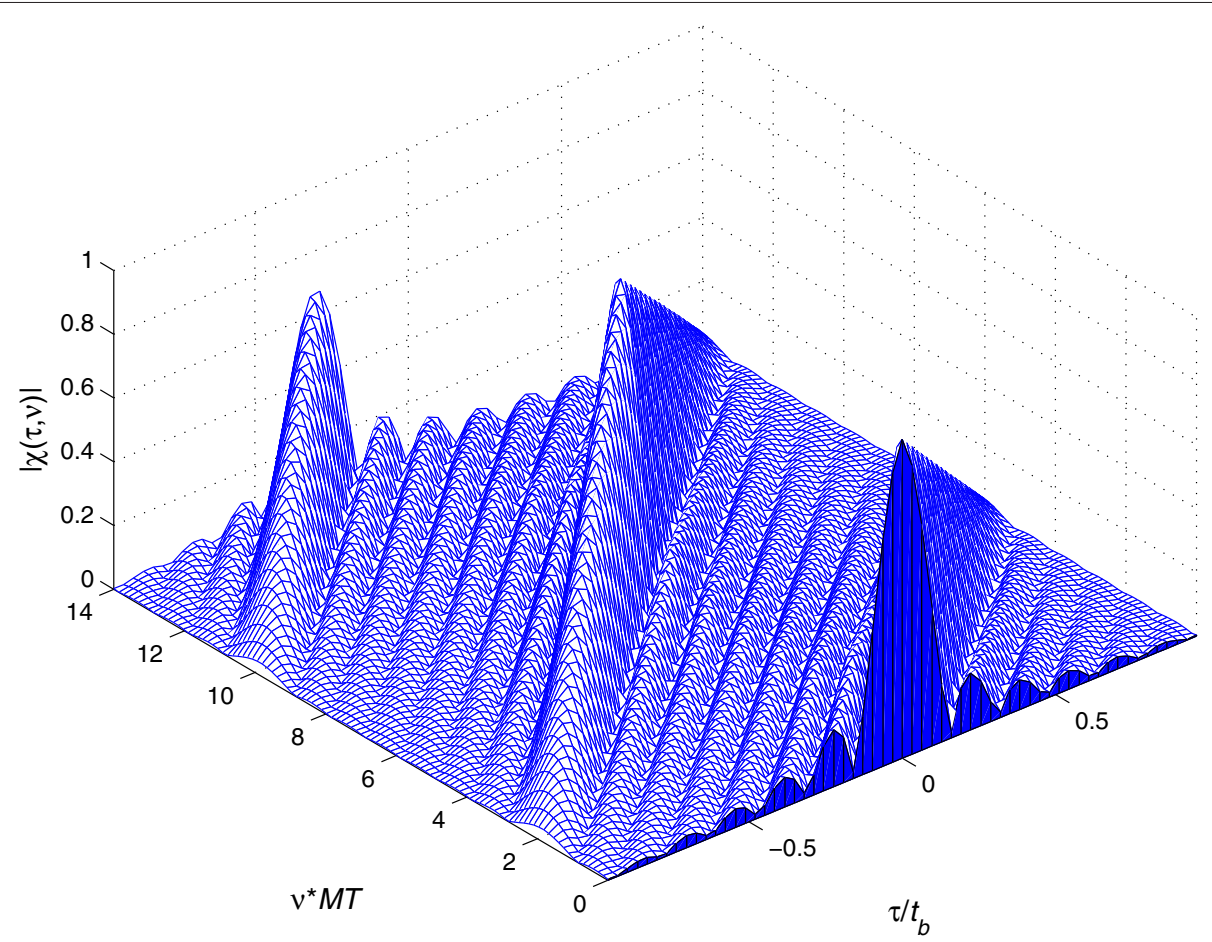

Figure 2 Ambiguity function of SF train of unmodulated pulses.

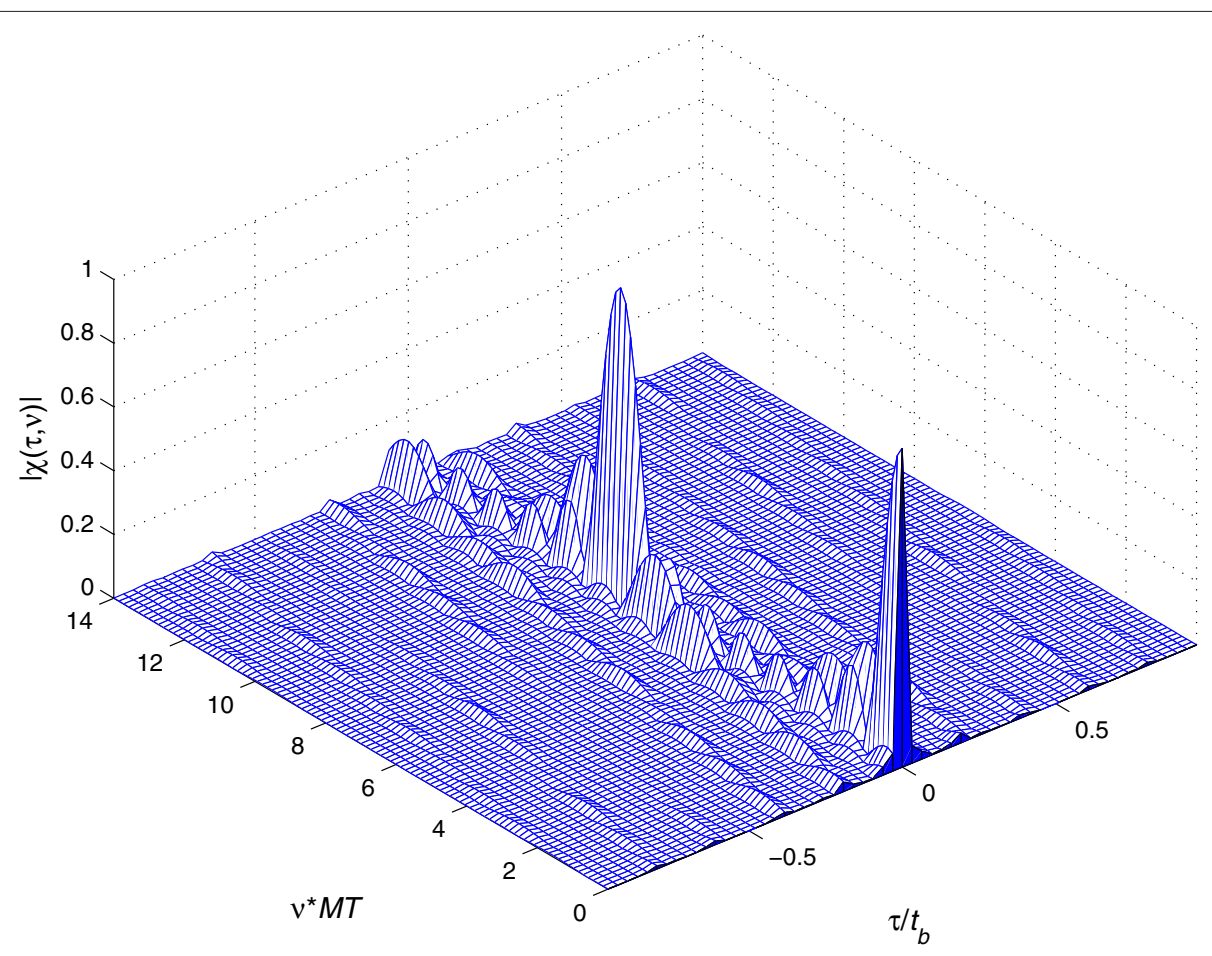

Figure 3 Ambiguity function of SF train of LFM. 
Assuming that $y_{n}$ are independent of each other, then the P.D.F. of $\mathbf{y}$ is

$$
f(\mathbf{y})=\prod_{n}^{N} f\left(y_{n}\right)=\prod_{n}^{N} \frac{y_{n}}{E^{2} \gamma^{2}+\sigma^{2}} \exp \left[-\frac{y_{n}^{2}}{2\left(E^{2} \gamma^{2}+\sigma^{2}\right)}\right]
$$

If $\theta \cong \gamma^{2}$, we can express (18) as

$$
f\left(y_{n}\right)=\frac{y_{n}}{E^{2} \theta+\sigma^{2}} \exp \left[-\frac{y_{n}^{2}}{2\left(E^{2} \theta+\sigma^{2}\right)}\right]
$$

Therefore, we represent the $\mathrm{ML}$ algorithm to estimate the RCS average value $\theta$ as

$$
\begin{aligned}
\widehat{\theta}_{M L}(\mathbf{y}) & =\arg \sup _{\theta \in R^{+}} f(\mathbf{y}) \\
& =\arg \sup _{\theta \in R^{+}} \prod_{n}^{N} \frac{y_{n}}{E^{2} \theta+\sigma^{2}} \exp \left[-\frac{y_{n}^{2}}{2\left(E^{2} \theta+\sigma^{2}\right)}\right]
\end{aligned}
$$

It is equivalent to maximize $\log f(\mathbf{y})$ (natural logarithm),

$$
\log f(\mathbf{y})=\sum_{n=1}^{N}\left[\log \left(\frac{y_{n}}{E^{2} \theta+\sigma^{2}}\right)-\frac{y_{n}^{2}}{2\left(E^{2} \theta+\sigma^{2}\right)}\right]
$$

It is a continuous function for $y_{n}>0$ and $\theta>0$, hence, a necessary condition for the ML estimation is

$$
\begin{aligned}
& \left.\frac{\partial}{\partial \theta} \log f(\mathbf{y})\right|_{\theta=\widehat{\theta}_{M L}(\mathbf{y})} \\
= & \frac{E^{2}\left(\sum_{n=1}^{N} y_{n}^{2}-2 N\left(E^{2} \theta+\sigma^{2}\right)\right)}{2\left(E^{2} \theta+\sigma^{2}\right)^{2}}=0
\end{aligned}
$$

Equation (24) has the unique solution

$$
\widehat{\theta}_{M L}(\mathbf{y})=\frac{1}{E^{2}}\left(\frac{\sum_{n=1}^{N} y_{n}^{2}}{2 N}-\sigma^{2}\right)
$$

Since $\theta>0$,

$$
\widehat{\theta}_{M L}(\mathbf{y})=\max \left[\frac{1}{E^{2}}\left(\frac{\sum_{n=1}^{N} y_{n}^{2}}{2 N}-\sigma^{2}\right), 0\right]
$$

Since

$$
\begin{aligned}
\left.\frac{\partial^{2}}{\partial \theta^{2}} \log f(\mathbf{y})\right|_{\theta=\widehat{\theta}_{M L}(\mathbf{y})} & =E^{4}\left(\frac{N}{\left(E^{2} \theta+\sigma^{2}\right)^{2}}-\frac{\sum_{n=1}^{N} y_{n}^{2}}{\left(E^{2} \theta+\sigma^{2}\right)^{3}}\right) \\
& =-\frac{4 E^{4} N^{3}}{\left(\sum_{n=1}^{N} y_{n}^{2}\right)^{2}} \\
& <0
\end{aligned}
$$

this solution gives the unique maximum of $\log f(\mathbf{y})$. The expectation of $\widehat{\theta}_{M L}(\mathbf{y})$ is then

$$
E_{\theta}\left[\widehat{\theta}_{M L}(\mathbf{y})\right]=E_{\theta}\left[\frac{\sum_{n=1}^{N} y_{n}^{2}}{2 N E^{2}}\right]-\frac{\sigma^{2}}{E^{2}}
$$

The mean value of $y_{n}$ is $\sqrt{\pi\left(E^{2} \theta+\sigma^{2}\right) / 2}$, and its variance is $(4-\pi)\left(E^{2} \theta+\sigma^{2}\right) / 2$. Since $y_{n}$ are independent of each other, it is

$$
E_{\theta}\left(y_{n}^{2}\right)=2\left(E^{2} \theta+\sigma^{2}\right)
$$

Therefore,

$$
\begin{aligned}
E_{\theta}\left[\widehat{\theta}_{M L}(\mathbf{y})\right] & =\frac{E_{\theta}\left[y_{n}^{2}\right]}{2 E^{2}}-\frac{\sigma^{2}}{E^{2}} \\
& =\theta
\end{aligned}
$$

As a result, it is an unbiased estimator.

Fisher's information [24] in this case can be obtained as

$$
\begin{aligned}
I_{\theta} & =-E_{\theta}\left[\frac{\partial^{2}}{\partial \theta^{2}} \log f(\mathbf{y})\right] \\
& =-E_{\theta}\left[\frac{N\left(E^{2} \theta+\sigma^{2}\right)-\sum_{n=1}^{N} y_{n}^{2}}{\left(E^{2} \theta+\sigma^{2}\right)^{3}}\right] \\
& =\frac{E^{4} N}{\left(E^{2} \theta+\sigma^{2}\right)^{3}}\left(E_{\theta}\left(y_{n}^{2}\right)-\left(E^{2} \theta+\sigma^{2}\right)\right) \\
& =\frac{E^{4} N}{\left(E^{2} \theta+\sigma^{2}\right)^{2}} .
\end{aligned}
$$

Taking Equation (28) into account, we can obtain the Cramer-Rao lower bound (CRLB) [24]

$$
\operatorname{Var}_{\theta}[\widehat{\theta}(\mathbf{y})] \geq \frac{1}{I_{\theta}}=\frac{\left(E^{2} \theta+\sigma^{2}\right)^{2}}{E^{4} N}
$$

From (31), we observe that CRLB is inversely proportional to the number of radars $N$ in the RSN, which means that the RSN with large $N$ will have a low CRLB. We draw this conclusion by assuming that the radar pulses are independent (in time and space) and follow a Rayleigh distribution, according to the 'Swerling 2' model.

\section{Simulation results}

\subsection{Signal recovery}

In this section, we study the scenario where we have $N$ transmit sensors but only one receiving sensor. We assume that the number of samples is set to 500. We apply the SF train of LFM pulses as pulse compression codes on the transmit side and use the CS technique in the receiving side. The classical 'Swerling model 2' and Gaussian noise are also considered in the simulation. The reconstructed signal is compared with the original signal by calculating the mean square error (MSE) in order to evaluate the reconstruction ability. The Mean Square Error between the original signal and the reconstructed signal is shown in Figures 4 and 5 as a function of different number of 


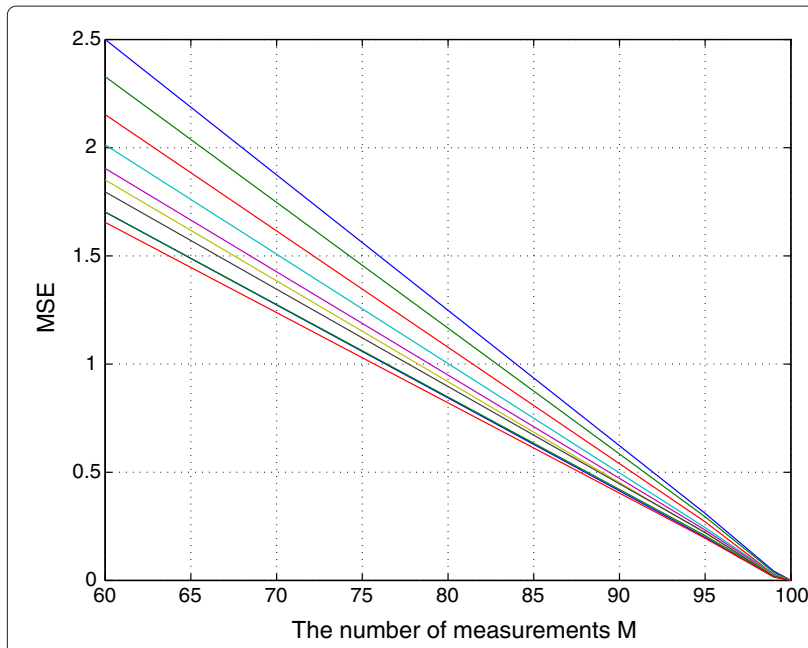

(a) Normalized MSE versus $M$. Each line corresponds to a different $\operatorname{SNR}(-5,4)$.

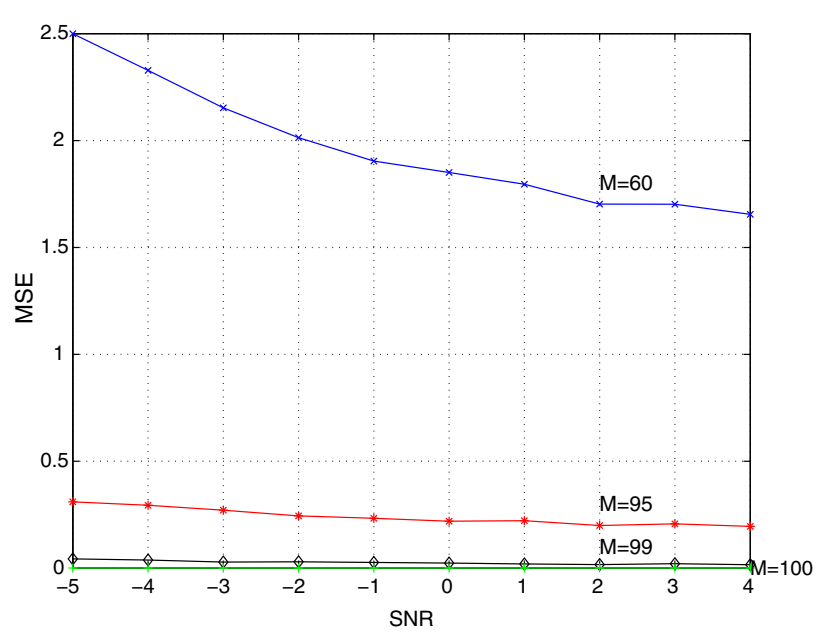

(b) Normalized MSE versus SNR. Each line corresponds to a different number of measurements $M$

Figure 4 Normalized MSE between reconstructed signal and original signal for fixed $\boldsymbol{N}=\mathbf{1 0 0}$. (a) Normalized MSE versuS $M$. Each line corresponds to a different SNR $(-5,4)$. (b) Normalized MSE versus SNR. Each line corresponds to a different number of measurements $M$.

measurements $M$ and SNR values. The noise considered here is introduced by the propagation in the air but not by compressing and decompressing process. We use the Monte-Carlo simulation model here and the results are averaged by $10^{5}$ runs/iterations. The cases of $N=50$ and $N=100$, where $N$ is the number of transmit sensors are illustrated in Figures 4 and 5, separately.

According to both Figures 4a and 5a, MSE is reduced as the number of measurements $M$ is increased. The system can perfectly reconstruct the signal which includes the received signal and the system noise when the number of measurements $M$ is equal to the number of transmit sensors $N$. In addition, the slope of MSE versus the number of measurements $M$ is almost a consistent for each $\mathrm{SNR}$ value. From Figures $4 \mathrm{~b}$ and $5 \mathrm{~b}$, we draw the same conclusion that the closer the number of measurements approaches $N(M \leq N)$, the better performance of signal recovery is achieved. In addition, we also discover that the MSE does not depend much on the SNR, especially when $M$ is large. As a result, the proposed model can be used

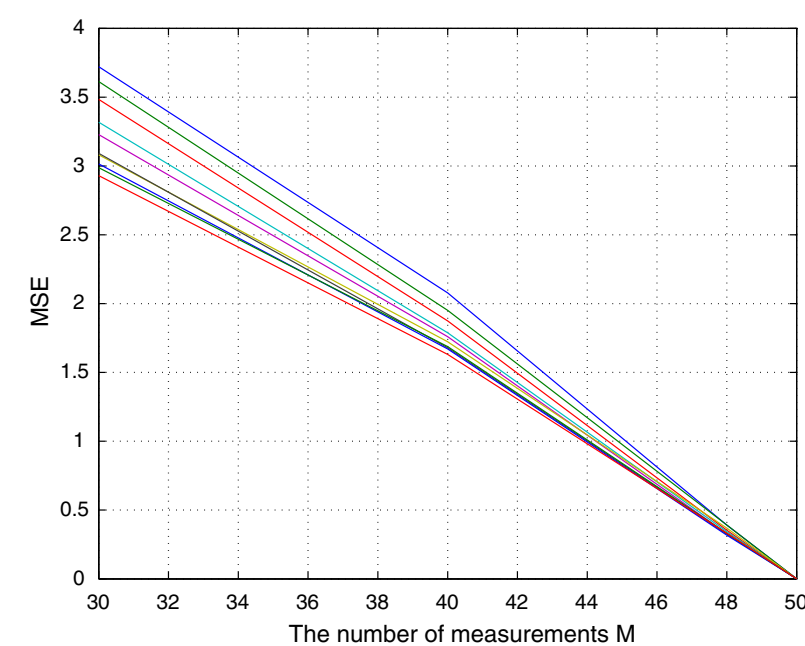

(a) Normalized MSE versus M. Each line corresponds to a different $\operatorname{SNR}(-5,4)$.

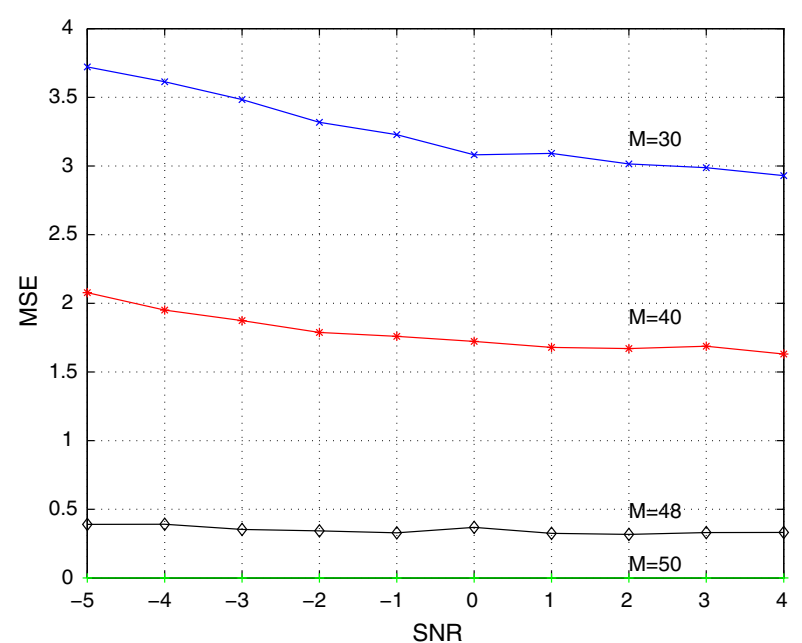

(b) Normalized MSE versus SNR. Each line corresponds to a different number of measurements $M$.

Figure 5 Normalized MSE between reconstructed signal and original signal for fixed $\boldsymbol{N}=\mathbf{5 0}$. (a) Normalized MSE versus $M$. Each line corresponds to a different SNR $(-5,4)$. (b) Normalized MSE versus SNR. Each line corresponds to a different number of measurements $M$. 
under a low SNR if the number of measurements $M$ could be properly chosen according to the number of transmit sensors $N$.

On the basis of the simulation results, we can draw a brief conclusion that the number of measurements $M$ of our model only depends on the size of RSN even when the number of samples is fixed as large as $\mathbf{5 0 0}$ here. Another important result emerging from the simulations is that the probability of target miss detection is zero no matter how small a number of measurements we use in the recovery process. That is to say, less measurements can be used to detect the target in the system, since a kind of diversity gain is achieved at the output of the matched filters on the receiving sensors.

\subsection{RCS parameter estimation}

In this section, we will consider the fluctuating target with an RCS parameter $\theta$ (following Rayleigh distribution) investigated in the Section 4. We will apply the ML estimation algorithm to estimate the parameter $\widehat{\theta}$. The scenario is similar to the one in the section above, but the number of samples in time domain is reduced to 100 for complexity reasons. We ran Monte Carlo simulations for $10^{5}$ iterations at each SNR value. We have considered the fluctuating target with RCS parameter $\theta=2$ (Small flighter aircraft or 4 passenger jet) in Figure 6. We plotted the variance of the RCS ML estimator with different number of radars in RSN.
According to Figure 6 , the variance of $\widehat{\theta}$ closely approaches the CRLB but doesn't exactly match it. The reason why the variance of $\widehat{\theta}$ is not exactly the same as the CRLB is that the noise included in Equation (10) cannot be termed as sparse as the transmit signal in the same sparsity matrix. Therefore, the noise which is considered in CRLB can not be perfectly reconstructed after the decompression. The power of noise $\sigma^{2}$ reduces so that the calculated CRLB might be lower than the practical one. As a result, a gap between the variance of $\widehat{\theta}$ and the CRLB. However, the gap between variance of $\widehat{\theta}$ and the CRLB is reduced by increasing the number of radars. It is also easy to see that the actual variance of $\widehat{\theta}$ reduces as the number of radars increases from $N=10$ to $N=20$. Hence, the actual variance of $\widehat{\theta}$ is inversely proportional to $N$, as we have shown in the theoretical result $\operatorname{Var}_{\theta}[\widehat{\theta}(\mathbf{y})] \geq \frac{\left(E^{2} \theta+\sigma^{2}\right)^{2}}{E^{4} N}$.

It is easy to see that the actual variance of $\widehat{\theta}$ and the CRLB do not change much as the SNR increases. Stating differently, our ML estimator performs well even for low SNR ratios. In all, the simulation results validate the theoretical results. The variance of the RCS parameter estimation satisfies the CRLB and our ML estimator on the RCS parameter is an accurate estimator.

\section{Conclusions}

Motivated by the representation of SF waveforms, we introduced CS to the RSN exploiting the pulse

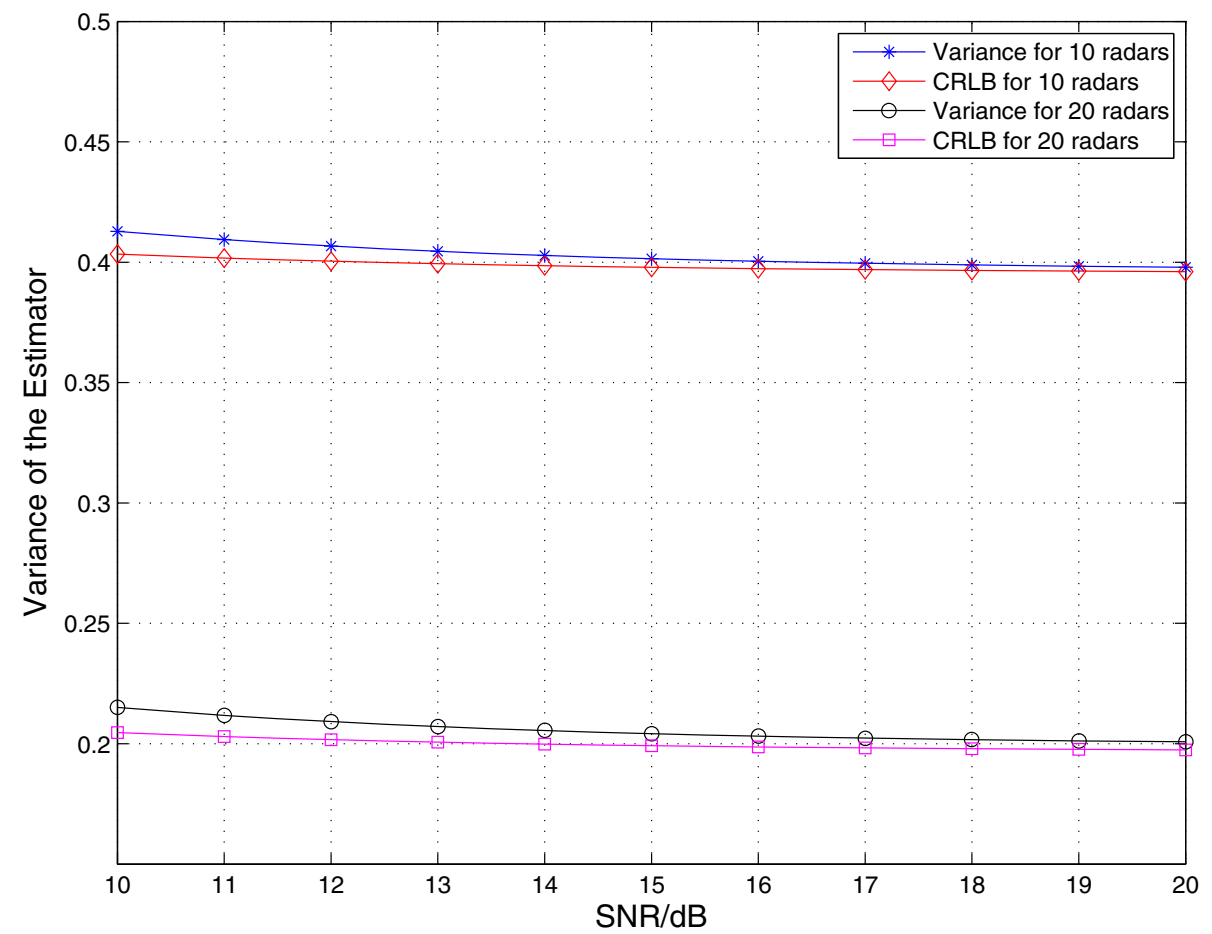

Figure 6 Variance of RCS ML estimator with different number of radars in RSN. The actual RCS parameter $\theta=2$. 
compression technique. A set of SF waveforms were applied as pulse compression codes at the transmit sensors, and the sparse matrix is also constructed based on the same SF waveforms. We observed that the signal samples along the time domain can be significantly compressed and recovered by using a small number of measurements which depend on the number of transmit sensors. A diversity gain is also achieved after the matched filters in the proposed model, so the probability of target miss detection can be zero even if the signal could not be perfectly recovered. In addition, we propose a ML algorithm to estimate the target RCS parameter and use the CRLB to successfully verify our theoretical result.

\section{Competing interests}

The authors declare that they have no competing interests.

\section{Acknowledgements}

This study was supported in part by National Science Foundation under Grants CNS-0964713, CNS-1017662, CNS-0963957, CNS-0964060, and Office of Naval Research under Grant N00014-11-1-0071.

\section{Author details}

${ }^{1}$ Department of Electrical Engineering, University of Texas at Arlington, Arlington, TX 76010, USA. ${ }^{2}$ Department of Computer Science, The George Washington University, Washington DC 20052, USA. ${ }^{3}$ Department of Preventive Medicine and Biometrics Uniformed Services, University of the Health Sciences Bethesda, Maryland 20814-4799, USA.

Received: 25 October 2012 Accepted: 5 January 2013

Published: 19 February 2013

\section{References}

1. S Mukhopadhyay, C Schurgers, D Panigrahi, S Dey, Model-based techniques for data reliability in wireless sensor networks. IEEE Trans. Mob. Comput. 8(4), 528-543 (2008)

2. T Onel, C Ersoy, H Delic, Information content-based sensor selection and transmission power adjustment for collaborative target tracking. IEEE Trans. Mob. Comput. 8(4), 1103-1116 (2009)

3. J Liang, Q Liang, Design and analysis of distributed radar sensor networks. IEEE Trans. Parallel Distrib. Process. 22(11), 1926-1933 (2011)

4. H Deng, Synthesis of binary sequences with good correlation and cross-correlation properties by simulated annealing. IEEE Trans. Aerosp. Electron. Systs. 8(8), 684-689 (2009)

5. Q Liang, Waveform design and diversity in radar sensor networks: theoretical analysis and application to automatic target recognition. IEEE Sensor Ad Hoc Commun. Netws. Conf. 2(28), 684-689 (2006)

6. Q Liang, Radar sensor networks for automatic target recognition with Delay-Doppler uncertainty. IEEE Military Commun. Conf. 23-25, 1-7 (2006)

7. MA Richards, Fundamentals of Radar Signal Processing (McGraw-Hill, 2005)

8. E Candes, M Walkin, An introduction to compressive sampling. IEEE Signal Process. Mag. 25(2), 21-30 (2008)

9. R Baraniuk, Compressive sensing. IEEE Signal Process. Mag. 24(4), 118-121 (2007)

10. E Candes, J Romberg, T Tao, Robust uncertainty principles: exact signal reconstruction from highly incomplete frequency information. IEEE Trans. Inf. Theory. 52(2), 489-509 (2006)

11. D Donoho, Compressed sensing. IEEE Trans. Inf. Theory. 52, 1289-1306 (2006)

12. E Candes, T Tao, Near-optimal signal recovery from random projections: universal encoding strategies. IEEE Trans. Inf. Theory. 52(12), 5406-5425 (2006)

13. R Baraniuk, P Steeghs, in Radar Conf., 2007 IEEE. Compressive radar imaging, (2007), pp. 128-133
14. AC Gurbuz, JH McClellan, WR Scott, in Proc. 41th Asilomar Conf. Signals, Syst. Comput. Compressive sensing for GPR imaging, Pacofoc Grove, CA, 2007), pp. 2223-2227

15. AC Gurbuz, JH McClellan, WR Scott, A compressive sensing data acqisition and imaging method for stepped frequency GPRs. IEEE Trans. Signal Process. 57, 2640-2650 (2009)

16. S Shah, Y Yu, A Petropulu, in 2010 IEEE International Conference on Acoustics Speech and Signal Processing (ICASSP). Step-frequency radar with compressive sampling (SFR-CS), (2010), pp. 1686-1689

17. Y Yu, AP Petropulu, HV Poor, MIMO radar using compressive sampling IEEE J. Sel. Top. Signal Process. 4(1), 146-163 (2010)

18. Y Yu, AP Petropulu, HV Poor, in 2010 4th International Symposium on Communications, Control and Signal Processing (ISCCSP). Range estimation for MIMO step-frequency radar with compressive sensing, (2010)

19. S Gogineni, A Nehorai, in 2010 International Waveform Diversity and Design Conference (WDD). Adaptive design for distributed $\mathrm{MIMO}$ radar using sparse modeling, (2010)

20. MA Herman, T Strohmer, High-resolution radar via compressed sensing. IEEE Trans. Signal Process. 57(6), 2275-2284 (2009)

21. P Swerling, Probability of detection for fluctuating targets. IRE Trans. Inf. Theory. 6, 269-308 (1960)

22. N Levanon, E Mozeson, Radar Signals (Wiley, New York, 2004)

23. Q Liang, X Cheng, KUPS: knowledge-based ubiquitous and persistent sensor networks for threat assessment. IEEE Trans. Aerosp. Electron. Syst. 44(3), 1060-1069 (2008)

24. JM Mendel, Lessons in Estimation Theory for Signal Processing, Communications, and Control (Prentice-Hall, Upper Saddle River, NJ, 1995)

doi:10.1186/1687-1499-2013-36

Cite this article as: $\mathrm{Xu}$ et al:: Compressive sensing in distributed radar sensor networks using pulse compression waveforms. EURASIP Journal on Wireless Communications and Networking 2013 2013:36.

\section{Submit your manuscript to a SpringerOpen ${ }^{\oplus}$ journal and benefit from:}

- Convenient online submission

- Rigorous peer review

- Immediate publication on acceptance

- Open access: articles freely available online

- High visibility within the field

Retaining the copyright to your article

Submit your next manuscript at $>$ springeropen.com 